\title{
Amplitude and Phase Mismatch Calibration Testbed for 2x2 Tower-Top Antenna Array System
}

\author{
Lukasz Lengier, Ronan Farrell \\ Centre for Telecommunications Value Chain Research (CTVR) \\ Institute of Microelectronics and Wireless Systems \\ National University of Ireland, Maynooth \\ Co. Kildare, Ireland \\ llengier@eeng.nuim.ie
}

\begin{abstract}
This paper aims to design a 2x2 antenna array testbed to serve as a complete tower-top calibration system that is able to eliminate the effect of transmitter differences of an array elements. The testbed architecture is based on the advanced tower-top interlinear reference calibration scheme where a non-radiative reference sensor is located in the centre of the array and four transmitter elements are coupled to its. The prototype system was built using commercial off the shelf components and it operates in the $2.4 \mathrm{GHz}$ ISM band. Software control capability supported with PC parallel port interface makes it flexible for various calibration algorithm implementation. A continuously updated (real time) calibration is also possible and easy to apply.

We presents an experimental results of tower-top calibration accuracy in transmit mode operation. The measured performance of prototype system calibration shows amplitude and phase imbalance better than $0.15 \mathrm{~dB}$ and $1.1^{\circ}$.
\end{abstract}

Keywords: array calibration, antenna array, base-station transmitter architecture

\section{Introduction}

In multiple antenna systems the output of individual elements of an array is spatially combined, aiming the array radiation in a specific direction. A given pattern directivity is equivalent to a given amplitude and phase relationship between each antenna element for particular frequency. In practical implementation of the antenna array systems these relationships can be distorted, causing inaccurate radiation pattern. The most significant influence on the magnitude of the phase and amplitude errors have distortions caused by differences between hardware associated to individual elements of an array system. These distortions might have origin from: finite manufacturing tolerance and ageing effect of components, the environmental factors such as pressure, humidity and, perhaps most significant temperature [1], and mutual coupling effects [2]. The presence of these many desperate sources of amplitude and phase imbalance necessitate some means of calibrating the array to ensure coherent combination of the radiated power.

Antenna array calibration issue has received considerable attenuation in recent years (for example [3 - 4]). These existing calibration procedures operate basis of a typical base transceiver station (BTS) that employs "tower-bottom" electronic equipment. However, as the alternative configuration for BTS where the RF portion of base station hardware is mounted directly to each element of an array at the tower-top (herein referred to as the "tower-top" architecture) is currently under investigation as novel tower-top antenna array calibration procedure is introduced by Cooper [ 5 
- 6]. This paper motivates a procedure that is used to calibrate our tower-top prototype testbed and proves the effectiveness of this approach.

Relocation RF hardware on the top of the tower offers both performance (the overall efficiency increase) and economic (the BTS capital cost reduction) potential benefits [7]. There are, however, many challenges associated with tower-top generation of transceiver design - high reliability, thermal management and reduction in size and weight. In order to meet the tower-top generation BTS requirements the digital IF transmitter architecture [8] is proposed as a one of the most promising and effective architectures.

This paper is organized as follows. Section 2 introduces tower-top calibration technique and briefly describes the particular case of transmit calibration scheme of 2x2 array. Section 3 presents proposed transmitter architecture, suitable for tower-top calibration. Section 4 gives details of our system implementation. Section 5 brings the calibration performance results. Finally section 6 presents our conclusions and discusses the future direction of work.

\section{Array Calibration}

\subsection{Tower-Top Calibration Scheme}

In a typical tower-top calibration scheme [5], showed in figure 1, the calibration is performed by feedback loop between each element of an antenna array and tower-top control unit where the errors are corrected. As a medium of the calibration feedback, such system usually employ transmission line paths - directional couplers [6] or power splitters [9]. Since any calibration signals are not transferred by free space, this method is referred as a non-radiative calibration. This technique allows to calibrate the effect of Tx an Rx differences of an array elements but not the antenna mutual coupling. This is important to notice also that the calibration accuracy is limited by the coupler amplitude and phase imbalances.

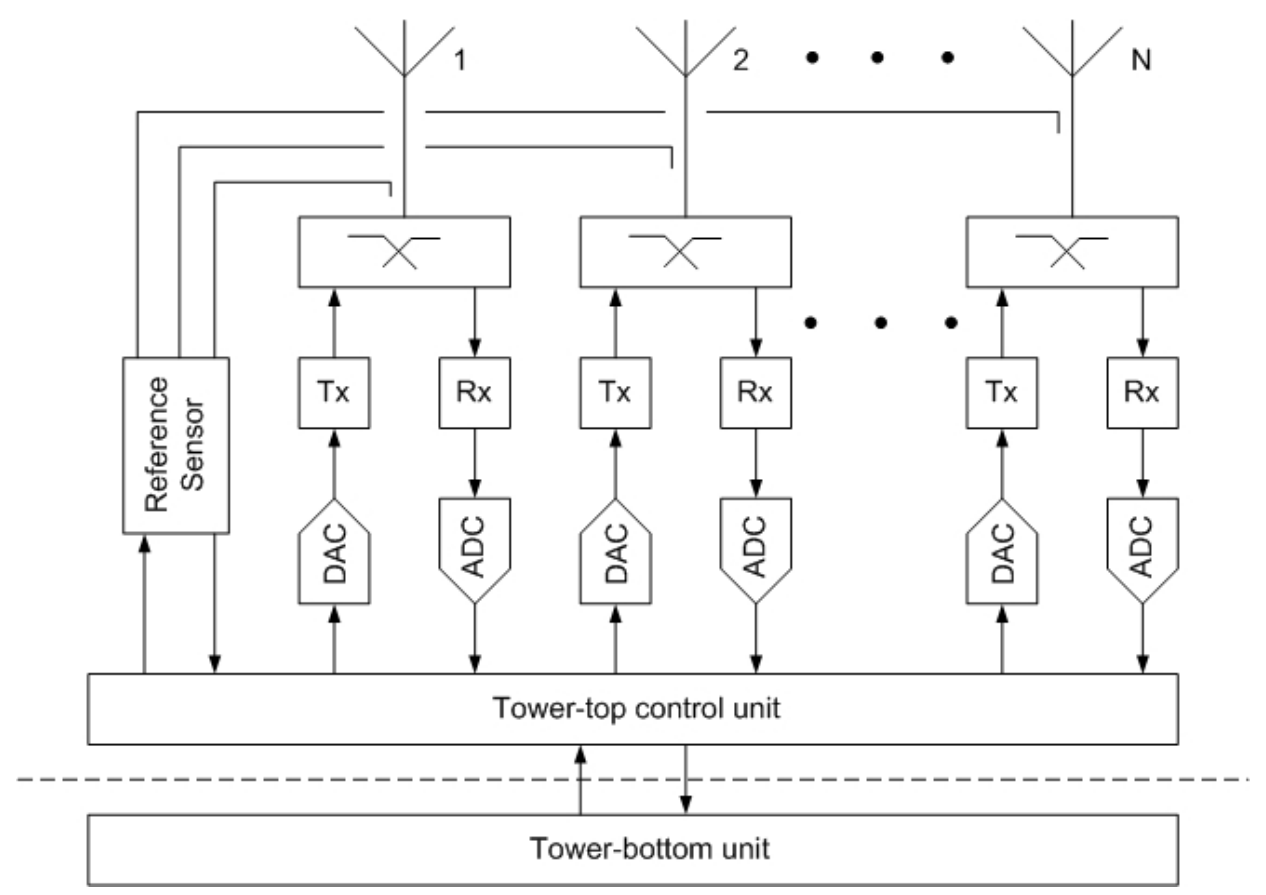

Figure 1. A typical tower-top calibration scheme.

The modulation/demodulation operations are performed by tower-bottom unit while the beamforming network is relocated on the top of the tower and its operations are executed in tower-top control unit. The transmitter and receiver chains associated to appropriate elements of the array are 
distributed between the tower-top control unit and antenna feed-points. Total calibration process is performed only by the tower-top circuitry.

This calibration scheme is versatile and might be employ to arrays of any geometrical configuration: linear, planar or circular. However, described approach brings significant challenge in design of suitable coupler arrangements. Moreover in the case of significant number of elements in the array, calibration becomes more complex and difficult to scaling.

\subsection{Transmit Calibration of a 2x2 Antenna Array}

The 2x2 antenna array calibration scheme [5-6], based on architecture from figure 1 , is presented in figure 2. In this case, a group of four transmitters is connected via calibration couplers network to reference sensor that is located in the centre of the array. In the case of transmit calibration mode, one of the transmitters is selected as a reference element (lets assume transmitter 1 ) and remaining transmitters are sequentially calibrated relative to its. The reference signal is fed back to the reference sensor where the reference phase and amplitude are measured and then stored in control unit. In next step, signal from transmitter 2 is transferred via coupler structure and measured. The difference between reference and current values defines the phase and amplitude errors. According to these errors, appropriate amplitude and phase correction is performed. The same operation is sequentially repeated for all of the Tx in the array. In result effective calibration of 2x2 array is achieved. The receive calibration is simply the reverse of the transmit calibration.

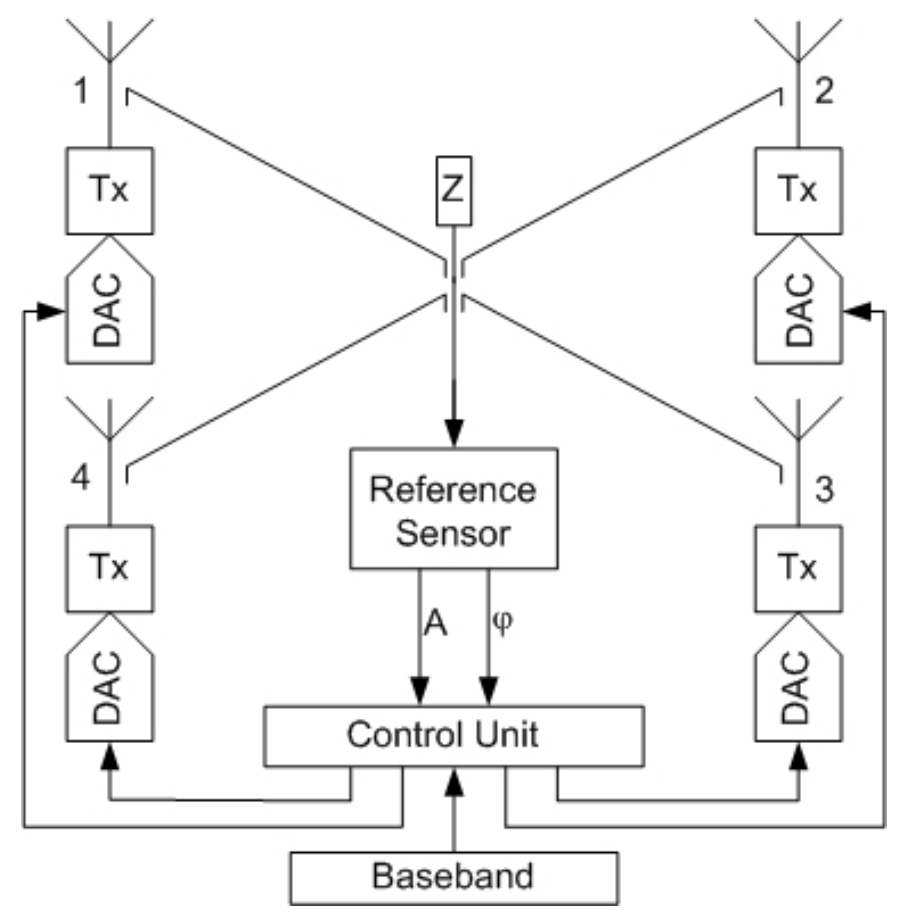

Figure 2. The calibration scheme of a 2x2 rectilinear antenna array with centered reference sensor.

\section{Transmitter Chain}

A key component of our proposed transmitter architecture (figure 3) is Direct Digital Synthesizer (DDS) that makes possible to perform first stage of up-conversion digitally. The digital operation increases the flexibility of the system due to output waveform parameters can be easily controlled by digital interface, while reducing size and cost. With the selected AD9954 [10] chip precise amplitude (resolution of 14 bits -0.0006 ASF granularity) and phase (resolution of 14 bits $-0.022^{\circ}$ granularity) adjusting, and consequently very accurate calibration can be achieved. Digital to analog converter that 
converts digital IF signal to analog is 14-bit, on-chip integrated, thus very high integration level is also achieved.

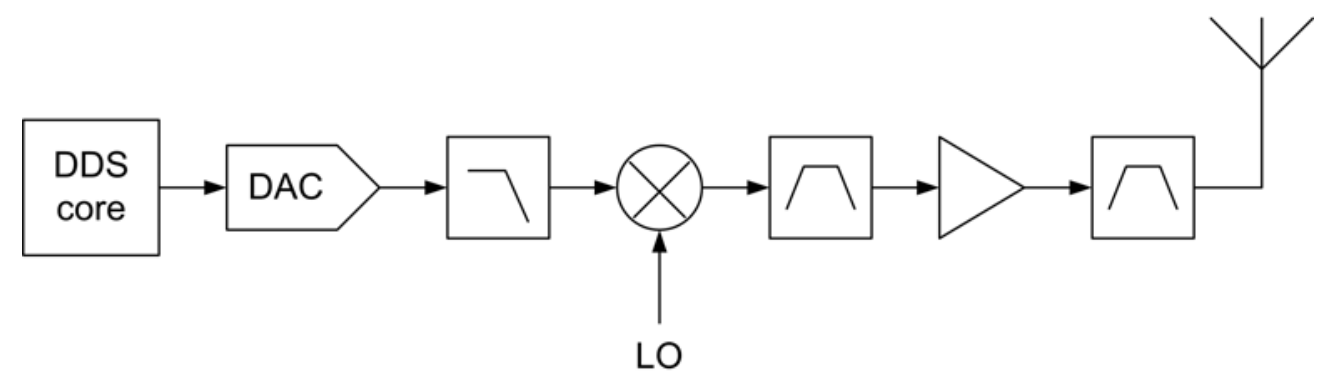

Figure 3. The conception of DDS with mixer up-conversion transmitter architecture.

The filtered DDS output is simply up-converted by mixer to RF frequency and then amplified. A general disadvantage of this approach is that the digital IF architecture still requires a significant amount of filtration at RF stage to overcome noise and spurious level which are higher then i.e. in direct transmitter architecture. This high filter requirements limits the integrity level significantly. However both RF filters are very low-cost due their microstrip implementation. Also external, high order IF filter is necessary to reject the DAC digital images which are located very close each other in the frequency range of desired signal (i.e. for desired $150 \mathrm{MHz}$ signal, first image frequency appears at $250 \mathrm{MHz}$ and power level only $5 \mathrm{~dB}$ down relative to carrier frequency). An inexpensive, small outline, nine order elliptic filter consists LC components was designed and implemented.

Special attenuation was paid to high reliability for tower-top transmitter operation. This is because the maintenance cost of a tower-top system in compared to one at the tower-bottom will be substantially higher due to the poor accessibility of hardware that is mounted at the top of a tower. The implication of this is a significant increase in cost if the reliability of the system is inadequate. Moreover, because such a system might be deployed in different locations around the world, the tower-top BTS will have to cope with extreme variations in its environment. It must be resistant to extremes of temperature and humidity, mechanical stress due to wind, heavy rain or ice and so on.

The power amplifier is the most critical components in the tower-top systems from high reliability point of view. Because of that the first attenuation should be paid in selecting the proper transistor with respect to high reliability performance and relatively low cost, i.e. ATF52189 [11] that features extremely high MTTF (over 300 years). Furthermore, the overall reliability prediction of designed amplifier board (according to The Military Reliability Handbook MIL-HDBK217 [12] count parts predictor method) for harsh environmental factor and $90 \%$ of confidence level is nearly 30 years and is comparable with current tower-top LNA performance [13].

For the purpose of calibration testbed system, the transmitter operates at $2.46 \mathrm{GHz}$ with a bandwidth of $40 \mathrm{MHz}$. For low-cost target, the FR4 substrate was employed to construct all of the circuitry (excluding both microwave band-pass filters) associated with this architecture and it was build using commercial off shelf components.

\section{System implementation}

The current antenna array calibration testbed operates only in transmit mode. The system architecture (figure 4) is based on the 2x2 calibration scheme from figure 2. The main features of the system blocks are listed below.

- The 2x2 calibration coupler board (based on six-port precision couplers [14]) that was invented by Cooper [6] is used in this testbed design.

- The transmitter chain, designed according to proposed architecture (section 3), was divided into two groups: RF (including parts following the mixer) and DDS (including also low-pass filter). Each individual RF transmitter circuitry was screened to avoid any possible coupling between adjacent chains and other electronic equipment. 
- For proper multi-DDS synchronization and clock distribution operations, a group of four DDSs is integrated on a common circuit board. This board is interfaced to a parallel port of PC control unit.

- A flexible control software, based on existing evaluation software source code [15] for AD9954, is written using Visual Basic 6 to perform multi-DDS operations and testbed system calibration. The current testbed software can perform only manual calibration. However, it can be easily configured to a real-time system that perform automatic calibration by developing existing software.

- the reference sensor element consists of phase and amplitude detector integrated on-chip. Nowadays, there exists some commercial off shelf component with adequate frequency range and accurate of amplitude and phase measurement scaling (i.e. AD8302 [16]). This circuitry is crucial from calibration accuracy point of view, that can be limited by finite phase and amplitude measurement accuracy. For example, in our testbed system the phase and amplitude measurements are displayed on scope which vertical resolution is limited to 8 bits, consequently deteriorates the calibration accuracy also to 8 bits.

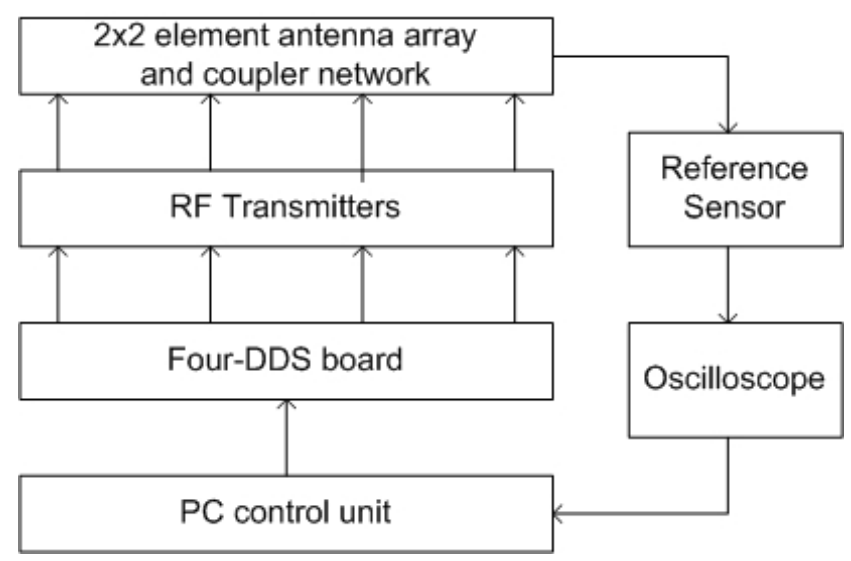

Figure 4. High-block testbed system architecture.

The current calibration testbed operates in manual mode of calibration. In first step the phase and amplitude measurements of the reference transmitter are taken. Then, in each calibration cycle, given transmitter is measured and then the amplitude and phase manually corrected (at the DDS stage) as long as the references values are obtained.

\section{$5 \quad$ Experimental Results}

The calibration testbed system configured as described in section 4 was used to investigate the calibration performance. Figure 5 shows a group of four output waveforms of the non-calibrated array testbed. There is maximum $0.313 \mathrm{~dB}$ amplitude and $67^{\circ}$ phase imbalances between two elements in the array. This illustrate the amplitude and especially phase distortion effects that might be introduced by hardware differences.

The successful calibration of the transmitted signals is illustrated in figure 6 . The amplitude calibration was performed first and the transmitters were calibrated relative to $\mathrm{CH} 3$ that characterizes the lowest output power. Then the phase calibration was processed, but this time the transmitters were calibrated relatively to $\mathrm{CH} 1$. The measured amplitude and phase imbalances of the calibrated testbed system equal to $0.1404 \mathrm{~dB}$ and $1.0689^{\circ}$ were obtained respectively. These results, fitted to a Gaussian probability function, are plotted on figure 7 and 8 , respectively.

\section{Conclusions}

In this paper we have presented a design of flexible tower-top antenna array calibration testbed system for testing the performance of variety calibration algorithms. The targets of low-cost, high reliability of the transmitter suitable for tower-top operation have been achieved. The low-cost of 
transmitter is crucial for antenna array system where the multiplication of hardware is significant. Furthermore, easy and flexible phase and amplitude control scheme based on DDS has been introduced. Also, the advantage is that the testbed system can be build using commercial off the shelf components.

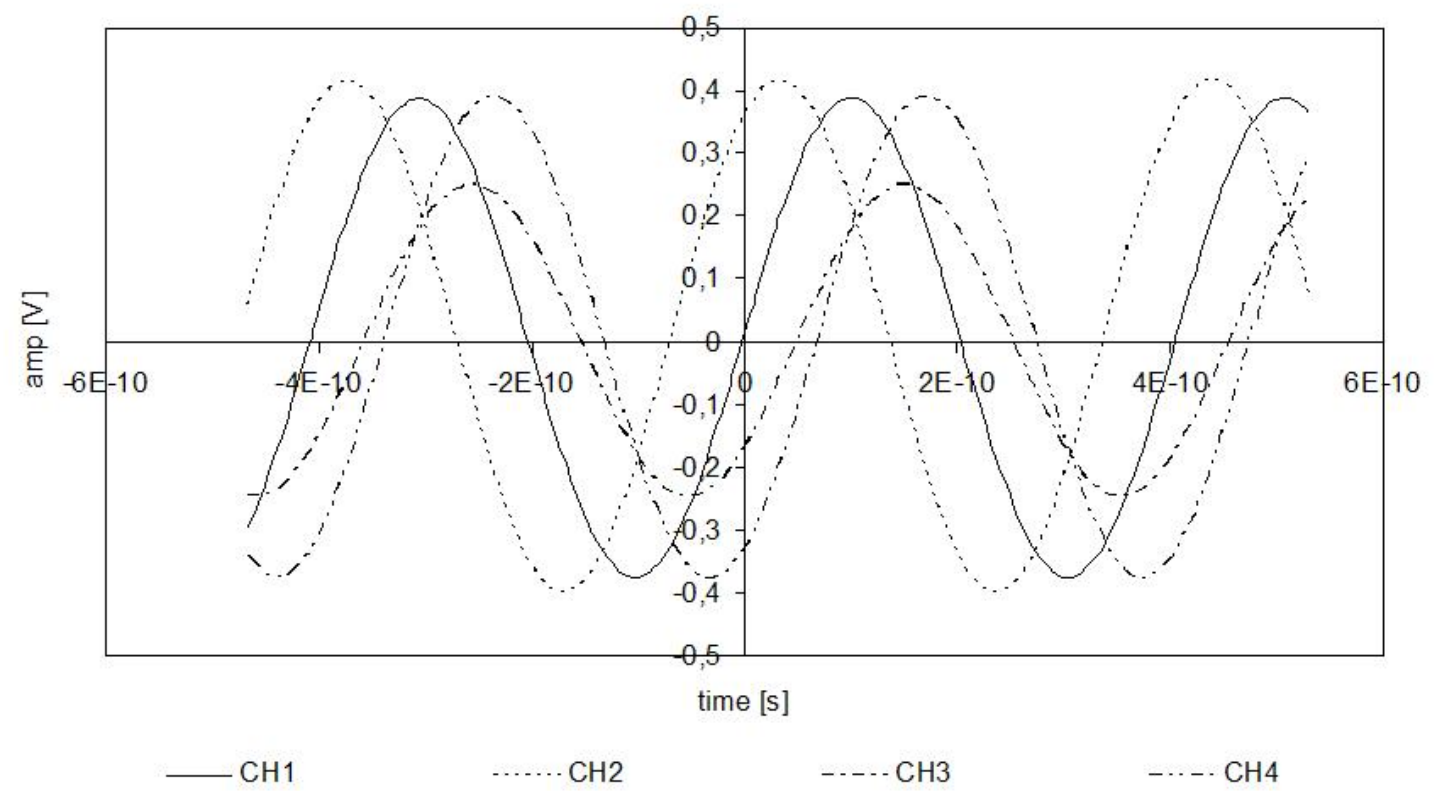

Figure 5. A group of output waveforms of the non-calibrated 2x2 antenna array testbed.

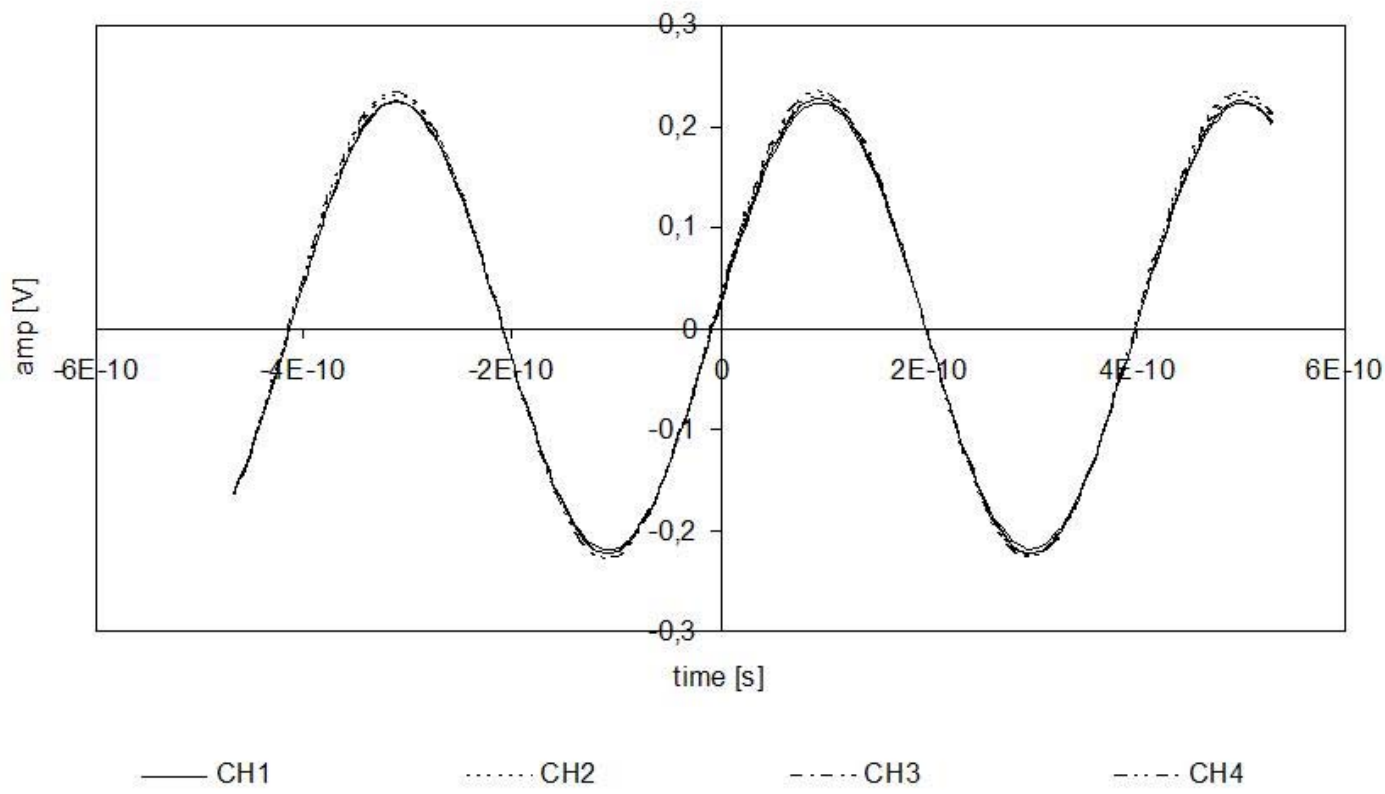

Figure 6. A group of output waveforms of the calibrated 2x2 antenna array testbed. 


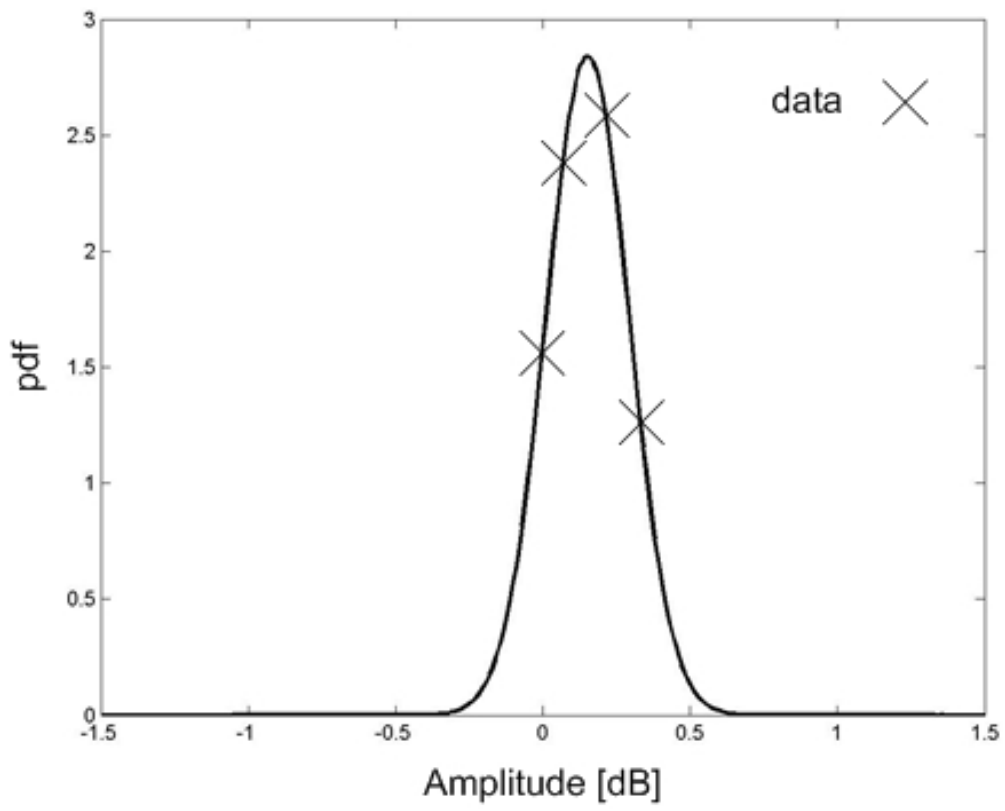

Figure 7. The measured amplitude imbalance of calibrated system, fitted to a Gaussian probability density function, $\mu_{A}=0.1517 \mathrm{~dB}, \sigma_{A}=0.1404 \mathrm{~dB}$.

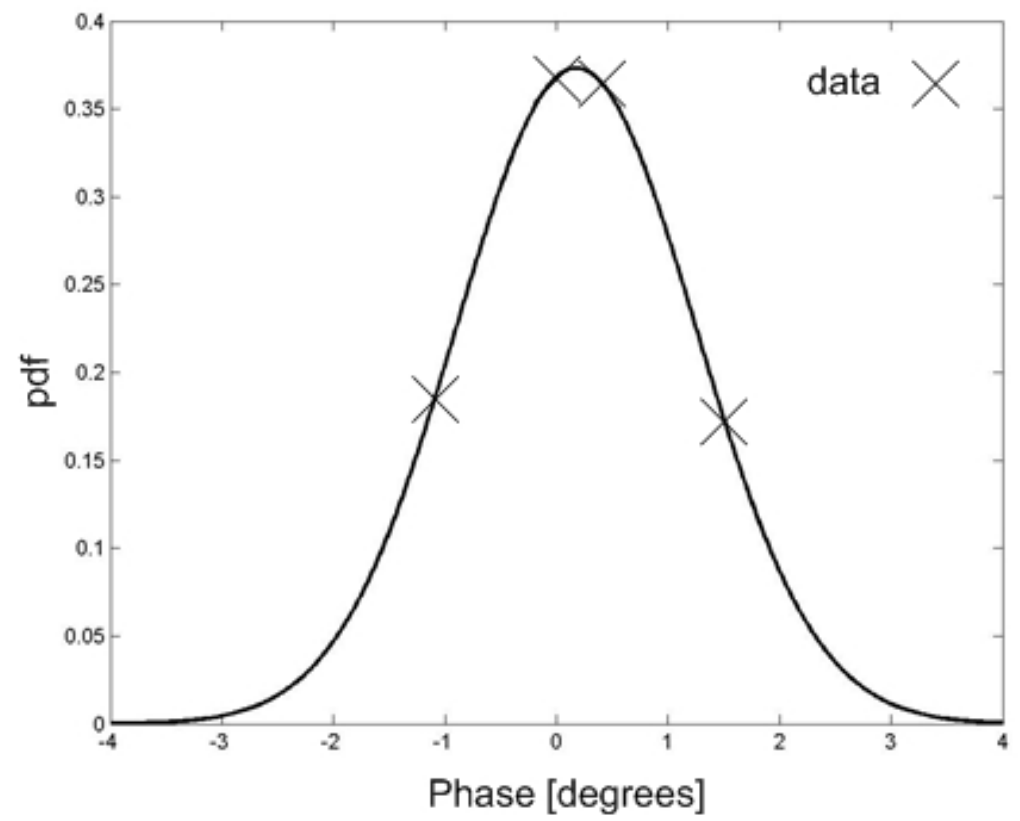

Figure 8. The measured phase imbalance of calibrated system, fitted to a Gaussian probability density function, $\mu_{\sigma}=0.1750^{\circ}, \sigma_{\sigma}=1.0689^{\circ}$.

Acknowledgements: The authors would like to thank Science Foundations Ireland (SFI) for their generous funding of this project through the Centre of Telecommunications Value Chain Research (CTVR). 


\section{References}

[1] Tyler, N.; Allen, B.; Aghvami, H. (2004) Adaptive Antennas: the Calibration Problem IEEE Communications Magazine, Volume 42, Issue 12, Dec. 2004, pages: 114-122

[2] Ozdemir, M.K.; Arslan, H.; Arvas, E. (2003) Mutual Coupling Effect in Multi-antenna Wireless Communication Systems Global Telecommunication Conference, GLOBECOM 2003, IEEE, Volume 2, 1-5 Dec. 2003, pages: 829-833

[3] Simmonds, C.M.; Beach, M.A. (1998) Downlink Calibration Requirements for the TSUNAMI (II) Adaptive Antenna Testbed The Ninth IEEE International Symposium on Personal, Indoor and Mobile Radio Communications, Volume 3, 8-11 Sept. 1998, pages: 1260-1264

[4] Dandekar, K.R.; Hao Ling; Guanghan Xu (2000) Smart Antenna Array Calibration Procedure Including Amplitude and Phase Mismatch and Mutual Coupling Effects IEEE International Conference on Personal Wireless Communications, 17-20 Dec. 2000, pages: 293-297

[5] Cooper, T.S.; McCormack, J.; Farrell, R.; Baldwin, G. (2007) Toward Scalable, Automated Tower-Top Phased Array Calibration Vehicular Technology Conference 2007, Dublin, Apr. 23-25 2007, pages: 5 pp.

[6] Cooper, T.S.; McCormack, J.; Farrell, R. (2007) Tower-Top Antenna Array Calibration Scheme for Next Generation Networks $6^{\text {th }}$ EURASIP Conference, 27-30 June 2007, pages: submitted

[7] Cooper, T.S., Farrell, R. (2007) Value-Chain Engineering of a Tower-Top Cellular Base Station System Vehicular Technology Conference 2007, Dublin, Apr. 23-25 2007, pages: 5 pp.

[8] Kermali, M.; Kumar, S.; LaSpisa, M.; Zhengxiang Ma; Martin, S.; Sreenath, K. (2002) Novel Base Station Radio Technologies for Third-Generation Wireless Applications Bell Labs Technical Journal, Volume 7, Issue 3, Autumn (Fall) 2002, pages: 151-167

[9] Xiao-le Yu; Da-ning Ni; Yun-xuan Wu; Fu-shun Zhang (2005) Design of a Calibration Network for Circular Smart Antenna Array Asia-Pacific Conference 2005, IEEE, Volume 3, 47 Dec. 2005, pages: 3 pp.

[10] Analog Devices datasheet (2007) 400 MSPS, 14-Bit, 1.8 V CMOS Direct Digital Synthesizer $A D 9954$ Analog Devices, Inc. 2007, pages: 1-40

[11] Agilent Technologies Datasheet Agilent (2005) ATF-52189 High Linearity Mode Enhancement Pseudomorphic HEMT in SOT89 Package 13 Sep. 2005, pages: 1-17

[12] MIL-HDBK-217F Military Handbook (1991) Reliability Prediction of the Electronic Equipment US Department of Defense, Washington DC, 2 Dec 1991, pages: 1-205

[13] ADC SMARTop Tower Top Amplifier technical documentation (2001) Target MTTF of DD1900 ADCP-75-103, Issue 3, March 2001, page 27

[14] Cooper, T.S.; Baldwin, G.; Farrell, R. (2006) Six-Port Precision Directional Coupler Electronic Letters, 12 Oct. 2006, Volume 42, Number 21, pages: 1232-1234

[15] Analog Devices Evaluation Boards/Tools (2003) AD9859, AD9951-AD9954 Evaluation Software Source Code, Rev 1.8 Analog Devices, Inc., 2002-2003

[16] Analog Devices datasheet (2002) LF-2.7 GHz RF/IF Gain and Phase Detector AD8302 Analog Devices, Inc., 2002, pages: 1-24 